\title{
Les cheminots dans la Résistance. Bibliographie thématique
}

Cécile Hochard

\section{OpenEdition}

\section{Journals}

Édition électronique

URL : https://journals.openedition.org/rhcf/540

DOI : $10.4000 /$ rhcf.540

\section{Éditeur}

Rails \& histoire

\section{Édition imprimée}

Date de publication : 1 juin 2006

Pagination : 115-148

ISSN : 0996-9403

Référence électronique

Cécile Hochard, "Les cheminots dans la Résistance. Bibliographie thématique », Revue d'histoire des chemins de fer [En ligne], 34 | 2006, mis en ligne le 16 mai 2011, consulté le 22 avril 2022. URL : http:// journals.openedition.org/rhcf/540 ; DOI : https://doi.org/10.4000/rhcf.540 


\section{Les cheminots dans la Résistance Bibliographie thématique}

\section{Cécile Hochard}

Docteur en histoire

\section{Méthode de travail adoptée pour l'élaboration et la présentation de cette bibliographie}

La bibliographie présentée ici, qui se veut la plus exhaustive possible pour les sources et travaux en langue française, a été élaborée dans le cadre de la préparation de l'exposition Les cheminots dans la Résistance. Elle présente les ouvrages et documents audiovisuels qui situent la résistance cheminote dans le contexte de l'occupation et de la Résistance en général, en tenant compte des avancées les plus récentes de la recherche en ce domaine et s'appuie sur des événements, des lieux, des parcours individuels et collectifs suffisamment divers pour justifier l'ambition nationale de l'exposition.

La bibliographie est présentée selon le plan suivant :

- les sources, au sens large ;

puis les ouvrages et articles sur:

- la SNCF avant guerre, ses activités pendant la guerre et l'occupation ;

- la Résistance : avec une sélection de travaux reflétant les acquis méthodologiques les plus récents de la recherche, ainsi que des monographies sur la Résistance, rapidement dépouillées pour les mentions des cheminots qu'elles contiennent;

- la résistance cheminote proprement dite ;

- la répression individuelle et collective contre les cheminots résistants ;

- la libération ;

- la mémoire de la résistance cheminote.

Les recherches bibliographiques ont été menées dans plusieurs lieux : à l'AHICF, au Fonds cheminot du Comité central d'entreprise de la SNCF, à l'Institut d'histoire du temps présent, au Mémorial Maréchal Leclerc de Hauteclocque et de la Libération de Paris - Musée Jean Moulin, à la Bibliothèque nationale de France et à la Fondation de la Résistance. Par ailleurs, des recherches ont été effectuées dans les catalogues en ligne de la Bibliothèque de documentation internationale contemporaine (BDIC), du Musée de la Résistance et de la Déportation de Besançon ainsi que sur la base des travaux universitaires de MER, mémoire et espoir de la Résistance (concernant la France sous l'Occupation). Les documents audiovisuels proviennent du Centre audiovisuel de la SNCF (CAV, certains étant en consultation à l'AHICF), de la BDIC, du Fonds cheminot du CCE SNCF, et des Archives du film à Bois-d'Arcy. 


\section{Instruments de travail, sources imprimées, témoignages, souvenirs}

\section{Instruments de travail}

Catalogue des tracts clandestins de la Deuxième Guerre mondiale conservés à la bibliothèque municipale de Toulouse. Originaux et photocopies, Toulouse, Bibliothèque municipale, 1975, $87 \mathrm{p}$.

Dictionnaire biographique du mouvement ouvrier francais (le Maitron), version CD-Rom, Les Éditions de l'atelier/Les Éditions ouvrières, 1997.

Résistance en Corse (La), CD-Rom édité par l'AERI (Association pour des études sur la Résistance intérieure), 2003.

Résistance dans l'Oise (La), CD-Rom édité par l'AERI, 2003.

Résistance dans l'Yonne (La), CD-Rom édité par l'AERI, 2004.

Résistance dans le Calvados (La), CD-Rom édité par l'AERI, 2004.

Résistance en Île-de-France (La), DVD-Rom édité par l'AERI, 2004.

Site internet de l'Ordre de la Libération [publie notamment des biographies des Compagnons].

Association nationale des médaillés de la Résistance française, Annuaire des médaillés de la Résistance française, tapuscrit.

BELTRAN (Alain) ; RUFFAT (Michèle), Culture d'entreprise et histoire, Paris, Les Éditions d'organisation, 1991, 158 p.

COINTET (Jean-Paul) ; COINTET (Michèle) (dir.), Dictionnaire historique de la France sous l'Occupation, Paris, Tallandier, 2000, 729 p.

GOERGEN (Marie-Louise) (dir.), Cheminots et militants. Un siècle de syndicalisme ferroviaire, Paris, Éditions de l'atelier/Éditions ouvrières, 2003, 431 p. [CD-Rom à paraittre.]

JAMMES (Pierre), «Essai de répertoire des tracts lancés par avion pendant la guerre 1939-1945», Le Vieux papier, n 135, p. 25-40 et n 136, p. 49-62.

KERBAUL (Eugène), 1270 militants du Finistère, 1918-1945. Dictionnaire biographique de militants ouvriers, Bagnolet, E. Kerbaul, 1985, 288 p.

KERBAUL (Eugène), 1485 militants du Finistère, 1918-1945. Cahier de mise à jour. Dictionnaire biographique de militants ouvriers, Bagnolet, E. Kerbaul, 1988, $64 \mathrm{p}$.

KERBAUL (Eugène), 1640 militants du Finistère, 1918- 1945. Cahier de mise à jour $n^{\circ}$ 2. Dictionnaire biographique de militants ouvriers, Bagnolet, E. Kerbaul, 1988, 64 p.

MARCOT (François) (dir.), avec la coll. de Bruno Leroux et de Christine Lévisse-Touzé, Dictionnaire historique de la Résistance, Paris, Robert Laffont, coll. « Bouquins », 2006, 1248 p. 
NOGUÈRES (Henri), et alii, Histoire de la Résistance en France de 1940 à 1945, 5 tomes, Paris, Robert Laffont, 1967-1981.

NOTIN (Jean-Christophe), 1061 compagnons. Histoire des Compagnons de la Libération, Paris, Perrin, 2000.

PERMEZEL (Bruno), Résistants à Lyon. 1144 noms, Lyon, Éditions BGA Permezel, 1992, 556 p.

PERMEZEL (Bruno), Résistants à Lyon. 1221 noms, Lyon, Éditions BGA Permezel, 1995, 655 p.

POUJOL (Jacques), Protestants dans la France en guerre (1939-1945). Dictionnaire thématique et biographique, Paris, Les Éditions de Paris, 2000, 301 p.

ROUX-FOUILLET (Paul); ROUX-FOUILLET (Renée), Catalogue des périodiques clandestins conservés à la Bibliothèque Nationale (1939-1945), Paris, Bibliothèque nationale, 1954, 282 p.

\section{Sources imprimées sur la SNCF et les cheminots}

À la mémoire des cheminots morts pour la France, 1951. [Référence trouvée à l'IHTP mais le document ne se trouve pas dans le fonds.]

"Aux cheminots morts pour la France », La Vie du rail, $\mathrm{n}^{\circ}$ spécial, 25 août 1964, 84 p.

"Les cheminots dans la Résistance, quarantième anniversaire de la Libération », La Vie du rail, numéro spécial, 1984. Édition remaniée du numéro du 25 août 1964, 84 p.

M. NARPS (dir.), La Région de l'Est de la SNCF de 1939 à 1945, Imprimerie des dernières nouvelles de Strasbourg, 1947, 215 p.

« Resistance and Reconstruction », French Railways, Londres, s.d., 20 p.

"Résistance aux chemins de fer français et la reconstruction », Railway Garette, no 12, 21 septembre 1945.

HARRANG (G.), À la découverte des chemins de fer et des cheminots, Paris, Les Éditions ouvrières, 1944, 151 p.

MARTY (André), L'Exécution et la victoire de Pierre Sémard, Casablanca, Impr. réunies, s.d., 12 p.

MARTY (André), Un de ceux grâce auxquels la France renaît : Pierre Sémard, Paris, Éditions du Parti communiste français, 1945, 14 p.

MARTY (André), Pierre Sémard, Paris, Éditions Norman Béthune, 1972, 15 p.

ODIC (Charles Jean), Cheminots de France, édité et vendu sous le patronage de «Résistance-Fer» au profit des familles de cheminots victimes de guerre, s.l., Éditions du tertre, 1952, 87 p.

Résistance-Fer, Bataille du Rail, 44 p. (non daté).

SNCF, Lexique français-allemand : termes usuels de chemins de fer, SNCF, 1940, $105 \mathrm{p}$. 
SNCF, Lexique technique ferroviaire français-allemand, allemand-français, SNCF, 1941, $300 \mathrm{p}$.

VRAUVAL (J.), Film de guerre : SMCF-SNCF, Paris, Imprimerie Nationale, 1947, 66 p.

Brochure relatant des épisodes de la guerre au sein des chemins de fer. Annexe comprenant des documents officiels.

Témoignages, souvenirs, entretiens, biographies

(par commodité de présentation on a réuni dans un seul ensemble les documents écrits et les documents audiovisuels, avec mention, pour ces derniers, du lieu de dépôt et de consultation)

50 entretiens réalisés dans le cadre des projets de recherche « Mémoires cheminotes, 1937-1948 » et « Mémoires cheminotes, 1948-1978» conservés par l'Association Repères et mémoires des mondes du travail (REMEMOT), 4, rue des Tours, 59000 Lille.

Association nationale des cheminots anciens combattants, Témoignages, 1939-1945, Paris, 1981, 65 p.

Cahiers Maurice Thorez, n 1 (avril 1966), interview de Lucien Midol par Claude Willard.

Cahiers de l'Institut d'histoire sociale CGT Cheminots, n 4 [paru à l'été 1999], " À propos des trains de la déportation», dossier coordonné par Pierre Vincent.

Contient notamment, les témoignages de Jean Fumeaux, membre des FTP de la région parisienne, p. 7-8, et d'Émile Jacubovicz, membre des FTP-MOI, p. 10-16.

Ceux du rail, documentaire de René Clément, 1942, sur la Côte-d'Azur, décrit la vie d'un mécanicien ; travellings qui montrent les casemates allemandes et italiennes sur la côte ; film envoyé à Londres. [Archives du film, Bois-d'Arcy.]

Ceux qui ont fait de la Résistance... vous parlent, Paris, France d'abord, 1992, $260 \mathrm{p}$.

Ouvrage collectif réalisé par l'ANACR comportant deux chapitres sur la résistance dans les chemins de fer (II : « La bataille du rail à Laroche Migennes », p. 35-100 et III : «La locomotive sabotée », p. 103-196).

Cheminots dans la bataille de France (Les), collection « Les dîners-débats de la Résistance », 2 cassettes audio. [BDIC.]

Contient des allocutions de Albert Guerville, André Aurousseau, Gaston Brucher, René La Combe, André Ségalat.

Dernier mot (Le), documentaire réalisé par Geneviève Joutard, produit par le CRDP de Besançon et l'Association des amis du Musée de la Résistance de Besançon, VHS, 52 mn. [Musée de la Résistance et de la Déportation de Besançon.]

Contient le témoignage de Jean Gautheron, cheminot à Dôle, résistant, chef de secteur FTP à Lons-le-Saulnier. 
Dernier témoin (Le), film de Jean-Marc Bourdet, 1991.

Entretien avec Gilbert Bonnet, cheminot résistant, 1994, FR3 - Île-de-France, 2 cassettes vidéo $(2 \times 20 \mathrm{mn})$ et une brochure. [BDIC.]

Rushes du film 1944-1994, l'été de la libertée, série diffusée du $1^{\text {er }}$ au 28 août 1994.

Entretien avec Marc Lefort, cheminot en 1944, 1994, FR3 - Île-de-France, 1 cassette vidéo $(2 \times 20 \mathrm{mn})$ et 1 brochure. [BDIC.]

Rushes du film 1944-1994, l'été de la liberté, série diffusée du $1^{\text {er }}$ au 28 août 1994 ; le témoin raconte ses souvenirs de cheminot durant la libération de Paris en août 1944.

Entretien avec Robert Quint, cheminot résistant, FR3 - Île-de-France, 1 cassette vidéo $(20 \mathrm{mn})$ et 1 brochure. [BDIC.]

Rushes du film 1944-1994, l'été de la liberté.

Pierre Sémard, héros de la Liberté (1945). [Archives du film, Bois-d'Arcy.]

Pilotes du Rail, 1947. [CAV.]

Renaissance du rail (La), 1944. [CAV.]

Résistants de fer, film de Pierre-François Didek, Paris, Zeaux Productions, La Chaîne histoire, 2002, VHS.

Souvenirs d'Ernest Lecam dit Néness, publié par le Comité d'établissement régional SNCF de Paris Saint-Lazare (années d’apprentissage 19331936 puis années de guerre).

Transcriptions, approuvées par les témoins, des entretiens suscités et réalisés par l'AHICF dans le cadre de la préparation du 8 colloque de l'AHICF, tapuscrit. [AHICF.]

AGNIEL (Charles), Les Laboureurs de la nuit, Montpellier, Éditions Causse - Graille - Castelnau, 1950, 185 p.

AGNIEL (Charles), Les Laboureurs de la nuit, Nice, Éditions La Lambrusque, 1968, 127 p.

ARMAND (Louis), Propos ferroviaires, Paris, Fayard, 1970, 246 p.

Association des amis de Louis Armand [Henri Malcor], Louis Armand. 40 ans au service des hommes, Paris, Charles Lavauzelle, 1986, 172 p.

BAGEARD (Jules), Résistant du quotidien (chauffeur), témoignage oral recueilli le 12 mai 2000, 1 cassette VHS $31 \mathrm{mn}$. [CAV, déposé également à l'AHICF.]

BECHILLON (Catherine de), Fille ainée de Henri Lang, directeur à la SNCF, déporté le 22 mars 1942, témoignage oral recueilli le 22 mai 2000, 1 cassette VHS, 1 h 10. [CAV, déposé également à l'AHICF.]

BERÇAÏTS (Julien), Résistant individuel, Bordeaux, témoignage oral recueilli le 17 mai 2000, 1 cassette VHS, 12 mn 34. [CAV, déposé également à l'AHICF.]

BERTHELOT (Jean), Sur les rails du pouvoir (1938-1942), Paris, Robert Laffont, 1968, $342 \mathrm{p}$.

BLAIRET (Pierre), Cheminot, Monaco, Éditions du Rocher, 1988, 224 p. 
BLANCHARD (Marcel), Cheminot victime du travail forcé (octobre 1942-mai 1945), témoignage oral recueilli le 4 mai 2000, 1 cassette VHS, 56 mn. [CAV, déposé également à l'AHICF.]

BOURDET (Claude), L'Aventure incertaine. De la Résistance à la Restauration, Paris, Stock, 1975, 479 p.

BRONCHART (Léon), Ouvrier et soldat. Un Français raconte sa vie, Vaisonla-Romaine, Imp. H. Meffre, 1969, 203 p.

BURGAUX (Ch.), Les Confidences d'Arsène. Souvenirs d'un chef de gare, Paris, Les Productions de Paris, 1959, $165 \mathrm{p}$.

CAMUSET (Maurice), La Vie d'un franc-tireur et partisan français, Ariel, matricule 3060, Sainte-Savine, SONODA, 1985, 352 p.

COURTET (Émile), Mémoires d'un cheminot maquisard du Cantal jusqu'à Lyon (mai à septembre 1944, Clermont-Ferrand, Impr. de J. de Bussac, 1946, $187 \mathrm{p}$.

DELEPAUT (Georges), «Du syndicalisme cheminot clandestin à la lutte armée dans les rangs FTPF », MEMOR (Mémoire de la Résistance en zone interdite), no 21-22 (1995), p. 57-68.

DELEPAUT (Georges), Entretien avec Yves Le Maner filmé le 22 juin 2004, 1 DVD, 2 h 02 mn [Saint-Omer, La Coupole.]

DESHAYES (Pierre), Entretien avec Yves Le Maner filmé le 16 juillet 2004, 1 DVD, 2 h 41 mn [Saint-Omer, La Coupole.]

DESPREZ (Édouard), Un cheminot raconte sa jeunesse, ses engagements : 1919 1949, Hellemmes, Association Repères et mémoires du monde du travail, 1998, 203 p.

Cheminot du Pas-de-Calais, prisonnier de guerre évadé, membre des FTPF et de la direction clandestine de la CGT, secrétaire du syndicat des cheminots de Lens à la Libération.

DILIGENT (André), Un cheminot sans importance, Paris, Éditions FranceEmpire, 1975, 253 p.

DUFOUR (André), Résistant FTP Limoges, témoignage oral recueilli le 15 mai 2000, 1 cassette VHS, 29 mn. [CAV, déposé également à l'AHICF.]

DUPREZ (Henri), "'Le train de Loos' ou 40 ans d'énigme », MEMOR (Mémoire de l'Occupation et de la Résistance en zone interdite), $\mathrm{n}^{\circ} \mathrm{G}$ (juin 1986), p. 49-64.

DURIX (Vincent), « Un résistant FTP jurassien "Nino" : Jean Gautheron », mémoire de maitrise d'histoire, sous la dir. de François Marcot, université de Besançon, 1992, 153 p.

EISENMANN (Jacques), Ingénieur des Ponts et Chaussées détaché à la SNCF, licencié en application de la loi interdisant la fonction publique aux fils d'étrangers en septembre 1940, témoignage oral recueilli en mai 2000, 1 cassette vidéo, 1 h 31 mn. [CAV, déposé également à l'AHICF.] 
FILET (Bernard), Il était une fois... mon évasion, s.l., L'Écho, 1997, 90 p. Évasion d'un camp de PG, retour à Brive où veut reprendre sa place de mécanicien à la SNCF, mais apprend par son chef de dépôt qu'il est condamné à mort par la Gestapo et que son nom est affiché dans le hall de la gare. Prend le maquis. Présenté comme un roman, mais histoire du père de l'auteur.

FLAMENT (Louis), Résistance-Fer Lyon, témoignage recueilli le 12 mai 2000, 1 cassette VHS, 26 mn. [CAV, déposé également à l'AHICF.]

GIRARD (Pierre), "La SNCF sous l'Occupation », in René de Chambrun et Josée Laval, La Vie de la France sous l'Occupation, Hoover Institute, Paris, Plon, 1957, p. 321-338.

Témoignages rassemblés par $\mathrm{M}$. et Mme René de Chambrun et déposés à la Fondation Hoover à partir de 1948. Cet extrait a été réimprimé dans : POLINO (MarieNoëlle) (dir.), «Les cheminots dans la guerre et l'occupation. Témoignages et récits", Revue d'bistoire des chemins de fer hors série, $7,2^{\mathrm{e}}$ éd. revue et augnmentée, Paris, AHICF, nov. 2004, p. 286-309.

GUÉDON (Gilbert), Résistant FTP Paris, témoignage oral recueilli le 16 mai 2000, 1 cassette VHS, 45 mn. [CAV, déposé également à l'AHICF.]

GUINGOUIN (Georges), Quatre ans de lutte sur le sol limousin, Paris, Hachette, 1974, $287 \mathrm{p}$.

HARDY (René), Le Livre de la colère, Paris, Robert Laffont, 1951, 209 p.

HARDY (René), Amère victoire (roman), Paris, Robert Laffont, 1955, 284 p.

HARDY (René), Derniers mots. Mémoires, Paris, Fayard, 1983, 572 p.

HEILBRONN (Max); VARIN (Jacques), Galeries Lafayette, Buchenwald, Galeries Lafayette, Paris, Economica, 1989, 177 p.

HERNIO (Robert), Témoignage sur la résistance dans les chemins de fer de la région parisienne, entretien réalisé par Serge Wolikow le 3 juin 1993, 1 cassette vidéo, $122 \mathrm{mn}$. [Fonds cheminot, CCE SNCF.]

HERNIO (Robert), Avant que les cloches sonnent, Montreuil-sous-Bois, Fédération CGT des cheminots, 2000, 284 p.

LADET (René), Ils ont refusé de subir. La Résistance en Drôme. (Mémoires d'un Corps franc et compagnie FFI). Notre Résistance, 1942-1944, Portes-lèsValence, Chez l'auteur, 1987, 391 p.

LADET (René), Résistant Portes-lès-Valence (Drôme) FFI, témoignage oral recueilli le 13 mai 2000, 1 cassette VHS, 42 mn. [CAV, déposé également àl'AHICF.]

LASSOU (René), Résistant FTP Limoges, témoignage oral recueilli le 17 mai 2000, 1 cassette VHS, $32 \mathrm{mn}$. [CAV, déposé également à l'AHICF.]

LAUPRÊTRE (Jean), Jean ou la fidélité d'un militant cheminot, s.l., Association des amis de Jean Lauprêtre, 1994. 
LE CHATELIER (Bernard), Matricule 51306, mémoires de déportation, récit, Paris, Les Éditions de la Bruyère, 1984, 192 p. (2 éd., 2005).

Ingénieur à la SNCF, correspondant du réseau Jade-Amicol et « boîte aux lettres » du groupe de résistance des cheminots de son établissement à Compiègne.

LE CORRE (Guy), Un cheminot rennais dans la Résistance, Paris, Éditions Tirésias / AERI, 2003, 67 p.

A appartenu au réseau Manipule.

LEGROS (René), Résistant FTP Limoges, témoignage oral recueilli le 15 mai 2000, 1 cassette VHS, 14 mn. [CAV, déposé également à l'AHICF.]

LÉMAN (Michel), Ombres et lumières : un moment d'histoire des cheminots de Paris-Nord, publié par le Comité d'établissement régional de la région SNCF de Paris-Nord.

LEPRÊTRE (Xavier), De la résistance à la déportation. Compiègne - Royallieu 1940-1944, Même au péril de la liberté - 2, Compiègne, 222 p.

LEO (Gerhard), Un Allemand dans la Résistance : un train pour Toulouse, Paris, Tirésias, 1997, 300 p.

Récit d'un jeune allemand de 19 ans, travaillant pour la Résistance sous l'uniforme de la Wehrmacht à la Transport Kommandantur de Toulouse. Autre édition : Paris, Messidor, 300 p. où les photos intérieures ne sont pas exactement les mêmes que dans celle-ci.

LÉVI (Bernard), Fils de Robert Lévi, directeur à la SNCF, exclu en application du statut des Juifs, lui-même résistant (réseau Gallia, Valence), témoignage oral recueilli le 19 mai 2000, 1 cassette VHS, 25 mn. [CAV, déposé également à l'AHICF.]

LÉVI (Bernard), X bis. Un juif à l'École polytechnique. Mémoires 1939-1945, Paris, Calmann-Lévy, 2005, 241 p.

LEYGUE (Marcel), Résistant FTP Limoges, témoignage oral recueilli le 15 mai 2000, 1 cassette VHS, $30 \mathrm{mn}$. [CAV, déposé également à l'AHICF.]

LINET (Roger), La Traversée de la Tourmente, 1933-1943, Paris, Messidor, 1990, 383 p.

p. 211-215 : sabotage à Épinay-sur-Seine, dans la nuit du 16 au 17 juillet 1941.

LUTAUD (Laurent), Lettres du train fantôme, Same Films production, 2002, $85 \mathrm{mn}, \mathrm{VHS}$. [AHICF.]

MIDOL (Lucien), La Voie que j'ai suivie. Un ingénieur au cour des batailles sociales (1900-1970), Paris, Éditions sociales, 1973, 221 p.

NAHAS (Gabriel), La filière du rail, Paris, France Empire, 1982, 243 p.

Histoire romancée d'un réseau d'étudiants organisant avec la Résistance une filière d'évasion en Espagne, octobre 1943.

OSTENDE (Jean-Pierre), Le Pré de Buffalo Bill. Mémoire collective des cheminots des ateliers du Prado, Marseille, Via Valeriano, 1990, 125 p. 
PASTORELLO (Jacques), "Lucien Michel Auguste Beysson, une vie de cheminot », Les Cahiers des Caisses de prévoyance et de retraite $S N C F, \mathrm{n}^{\circ} 6$ (juin 2004), p. 125-140.

PATIN (Pierre), Rail et pavés. Paris, août 1944, récit d'événements vécus, Paris, La Pensée universelle, 1994, 142 p.

Ingénieur à la $\mathrm{SNCF}$, a tenu son journal depuis le débarquement.

PÉROCHE (Marcel), Les Mémoires de Marcel Péroche, "s sénateur 》 du rail, Paris, Berger-Levrault, 1984, 256 p.

PÉROCHE (Marcel), Ne touche pas à la locomotive! Scènes de la vie de Marcel Péroche, ancien mécanicien de l'Orient-Express, Paris, France-Empire, 1990, $205 \mathrm{p}$.

POLINO (Marie-Noëlle) (dir.), « Les cheminots dans la guerre et l'occupation. Témoignages et récits », Revue d'histoire des chemins de fer hors série, 7, $2^{\mathrm{e}}$ éd. revue et augmentée, Paris, AHICF, nov. 2004, 326 p.

RENOUF (Fernand), Cheminot victime du travail forcé (octobre 1942-mai 1945), témoignage oral recueilli le 4 mai 2000, 1 cassette VHS, 56 mn. [CAV, déposé également à l'AHICF.]

ROSSET (Georges), Quelques-uns des souvenirs, racontars, histoires d'un cheminot en retraite, Cannes, Refuge des cheminots de Campestra, 1951, 52 p.

RUELLE (André), L'Ombre couvrait l'effort et le soleil se leva. Journal d'un cheminot résistant de septembre 1940 à septembre 1944, s.l., Éditions Les Bruits de la nuit, 1979, $135 \mathrm{p}$.

SOUCHE (Yvette), Résistance-Fer Lyon, témoignage oral recueilli en janvier 2000, 1 cassette VHS, 58 mn. [CAV, déposé également à l'AHICF.]

SUDREAU (Pierre), Au-delà de toutes les frontières, Paris, Odile Jacob, 1991, $367 \mathrm{p}$.

THIÉRY (Christian), Cheminots en guerre, cheminots en paix. Souvenirs et anecdotes des cheminots de Blainville-D amelevières. Première partie, 1997, Mairie de Damelevières (54), 283 p.

THIÉRY (Christian), Cheminots en guerre, cheminots en paix. Souvenirs et anecdotes des cheminots de Blainville-Damelevières. Deuxième partie, 2001, Mairie de Damelevières (54), 495 p.

TONNAIRE (Jacques), Souvenirs d'un mécano de locomotive, 1932-1950, Paris, Jean-Claude Lattès, 1982, 235 p.

TOURNOIS (Yvon), Résistant FTP Limoges, témoignage oral recueilli le 15 mai 2000, 1 cassette VHS, 7 mn. [CAV, déposé également à l'AHICF.]

WOLKOWITSCH (Maurice), "Témoignage : résistance armée et chemins de fer », in "Armées et chemins de fer », Revue d'histoire des chemins de fer, 15 (automne 1996), p. 251-253. 
TRIAI (Raymond), « Rail contre fusées. Guerre 1939-1945. Témoignage. Nice, mai 1990 », in Michel El Baze, Les Guerres du XX siècle à travers les témoignages oraux, CD-Rom mis en ligne sur Internet (http://war.megabaze.com).

VINCENOT (Henri), Mémoires d'un enfant du rail : le rempart de la Miséricorde, Paris, Hachette littérature, 1980, 392 p.

Sur l'entre-deux-guerres (roman).

\section{L'entreprise et les métiers avant guerre - les transfor- mations induites par la guerre et I'occupation}

AHICF, Une entreprise publique dans la guerre. La SNCF, 1939-1945, Paris, PUF, 2001, 414 p.

Actes complets du colloque réuni à l'Assemblée nationale les 21 et 22 juin 2000. Les textes sans les débats qui les ont suivis sont en ligne sur le site : www.ahicf.com.

ALTWEGG (Jürg), L’Odyssée du train fantôme. 3 juillet 1944 : une page de notre histoire, Paris, Robert Laffont, 2003, 297 p.

ARMAND (Louis), Propos ferroviaires, Paris, Fayard, 1970, 246 p. Chap. 1 et 2, p. 19-74.

BACHELIER (Christian), « La SNCF sous l'Occupation allemande, 19401944, Rapport documentaire », Paris, IHTP-CNRS, 1996, 914 p. de textes et 2 volumes d'annexes.

Les textes sans les annexes documentaires sont en ligne sur le site : www.ahicf.com.

BACHELOT (Bruno), "La mise en place de la législation anticommuniste à la SNCF, de septembre 1939 à novembre 1942 », mémoire de maittrise d'histoire, sous la dir. de Nadine Vivier, université du Maine, septembre 2002, 217 p. [Déposé à l'AHICF.]

BAROLI (Marc), Les Cheminots, Paris, Éditions Atlas, 1987, 223 p.

BAUDOUI (Rémi), Raoul Dautry, 1880-1951. Le technocrate de la République, Paris, Balland, 1992, 398 p.

BELTRAN (Alain) ; FRANCK (Robert) ; ROUSSO (Henry), La Vie des entreprises sous l'occupation, Paris, Belin, 1994, 457 p.

BOCQUILLON (Alain) ; BRÉEMERSCH (Pascale); GHIENNE (Bernard), Lens, la gare, le dépôt, la cité des cheminots, Les dossiers de Gauhéria, $\mathrm{n}^{\circ}$ 5, 1996, $257 \mathrm{p}$.

p. 105-129 : sur les cheminots pendant l'occupation et la Résistance, sur les bombardements de l'été 1944.

BROUDER (Annie), Les Cheminotes, Paris, L'Harmattan, 1997, 247 p.

CARON (François), Histoire des chemins de fer en France, tome second, 1883 1937, Paris, Fayard, 2005, 1029 p. 
CARRIÈRE (Bruno), « 27 mars 1942, le premier convoi de déportés », La Vie du rail, $\mathrm{n}^{\circ} 2346$ (23 mai 1992), p. 27-32.

CARTER (Ernest F.), Les Chemins de fer en guerre, Paris, Presses de la Cité, $1965,250 \mathrm{p}$.

CATTIN (Étienne), Trains en détresse, Paris, Julliard, 1954, 231 p.

CHEVANDIER (Christian), "Bœufs et voituriers : les travailleurs des ateliers SNCF d'Oullins (1938-1947) », mémoire de maitrise d'histoire, sous la dir. d'Yves Lequin, université Lumière - Lyon II, 1986, 339 p. [Déposé à l'AHICF.]

CHEVANDIER (Christian), « Clivages et continuité dans les perceptions et les comportements ouvriers : les Ateliers d'Oullins de la SNCF ", in Denis Peschanski et Jean-Louis Robert (dir.), Les Ouvriers en France pendant la Deuxième Guerre mondiale, Actes du colloque des 22-24 octobre 1992, Paris, Centre d'histoire des mouvements sociaux et du syndicalisme / université Paris I / IHTP-CNRS, 1992, p. 431-441.

CHEVANDIER (Christian); FUKASAWA (Atsushi) ; RIBEILL (Georges), "Le statut des cheminots : genèse, historique et représentations ", communication présentée au colloque Professions et réglementations des transports dans la perspective européenne 1993, Paris, 9-11 mai 1989, 38 p.

CHEVANDIER (Christian); SARAZIN (Anne), Les Cheminots de la Région de Lyon pendant la guerre, catalogue de l'exposition présentée dans les gares et sites ferroviaires de la région lyonnaise, printemps 1995, au Centre d'histoire de la Résistance et de la Déportation, Lyon, hiver 1996.

COGNASSON (Patrick), Cheminots champenois. Romilly-sur-Seine : les ateliers de l'Est, Amiens, Martelle, 2000, 181 p.

p. 94-115: « Les ateliers pendant la Seconde Guerre mondiale » avec notamment le groupe FTPF de Maurice Camuset qui a réalisé des opérations de sabotage dans les Ateliers.

CONAN (Éric), «1940-1944, la SNCF sous la botte », L'Express, 4-10 février 1999, p. 90-94.

CRÉMIEUX-BRILHAC (Jean-Louis), Les Français de l'an 40, tome II, Ouvriers et soldats, Paris, Gallimard, 1990, 740 p.

DARD (Olivier) ; DAUMAS (Jean-Claude); MARCOT (François) (dir.), L'Occupation, l'État français et les entreprises, actes du colloque organisé par l'université de Franche-Comté, le Musée de la Résistance et de la Déportation de Besançon, 24-26 mars 1999, Paris, ADHE, 2000, 487 p.

DURAND (Paul), "La politique de l'emploi de la SNCF pendant la Deuxième Guerre mondiale », Revue d'histoire de la Deuxième Guerre mondiale, $\mathrm{n}^{\circ} 57$ (janvier 1965), p. 19-40. 
FIASSON (Roger), De mémoires de guerre en mémoires de gares, Saint-Étienne, Éditions des Arts Graphiques, 2002, 232 p.

FLORENTIN (Eddy), Quand les Alliés bombardaient la France, 1940-1945, Paris, Perrin, 1997, 476 p.

p. 265-279 : «L'autre bataille du rail» et p. 280-305: « 27 raids de la RAF sur 18 triages français. »

FLORES (Mariano), L'Étoile de Veynes, Grenoble, Presses et éditions ferroviaires, 1999, $320 \mathrm{p}$.

FOUANON (Arnaud), "Les cheminots de Laroche-Migennes; de la nationalisation aux grèves de 1947 », mémoire de maitrise d'histoire, université de Bourgogne, 1997.

FORTHOFFER (Joël), « La SNCF en Alsace et Moselle de 1939 à 1945 : le rôle des transports ferroviaires ", in $\mathrm{AHICF}$, Une entreprise publique dans la guerre. La SNCF, 1939-1945, Paris, PUF, 2001, p. 195-204.

FORTHOFFER (Joël), "La résistance des cheminots en zone annexée », in Robert Vandenbussche (dir.), Les Services publics et la Résistance en zone interdite et en Belgique (1940-1944), Actes du colloque de Bondues, 30 janvier 2004, Centre de recherche sur l'histoire de l'Europe du NordOuest / CEGES / université Charles-de-Gaulle-Lille 3, 2005, p. 83-99.

FROMENTIN (Natacha), «La gare d'Amiens et le réseau amiénois, 19391947 », mémoire de maîtrise d'histoire, sous la dir. de Michel-Pierre Chélini, université de Picardie, 2000, 149 p. [Déposé à l'AHICF.]

FROMENTIN (Natacha), «La vie quotidienne des cheminots des gares d'Amiens-Longueau pendant la guerre : bombardements, sabotages et reconstruction », in AHICF, Une entreprise publique dans la guerre. La SNCF, 1939-1945, Paris, PUF, 2001, p. 241-247.

GABORIAU (Patrick), Un village cheminot des Hautes-Alpes, septembre 1994, $110 \mathrm{p}$.

GERMAIN (Nicolas), "Les chemins de fer bas-normands pendant la Deuxième Guerre mondiale », mémoire de maitrise d'histoire, université de Caen, 1995.

GROUPE ARCHIVES QUATRE-MARES, Les Ateliers de Quatre-Mares. Huit décennies au service de la réparation des locomotives, Paris, Éditions La Vie du rail, 1996, $311 \mathrm{p}$.

Ouvrage illustré de nombreuses photographies. Quatre-Mares est un atelier de réparation du matériel de la SNCF situé sur les communes de Saint-Étienne-duRouvray et de Sotteville-lès-Rouen. p. 125-174 : chapitre V : "Quatre-Mares pendant la guerre (1939-1947). »

GUCKES (Jochen), « Le rôle des chemins de fer dans la déportation des juifs de France », Revue d'histoire de la Shoah. Le Monde juif, n 165 (janvier-avril 1999), p. 29-110. 
GUIBERT (Fabien), « Le détachement de main-d'œuvre de la SNCF aux chemins de fer allemands (1942-1944)», mémoire de maitrise d'histoire, sous la dir. de Nadine Vivier, université du Maine, septembre 2002, 175 p. [Déposé à l'AHICF.]

Institut CGT d'histoire sociale Rhône-Alpes, Grandes entreprises de la région Rhône-Alpes dans la Deuxième Guerre mondiale. Collaboration économique et résistance dans les entreprises, Actes du colloque, Lyon, 27 novembre 2001, 71 p. +12 p. d'annexes.

Voir l'intervention de Pierre Vincent sur la SNCF.

KALMBACHER (Jean) (dir.), "Le statut des chemins de fer français et leurs rapports avec l'État, 1908-1982», Revue d'histoire des chemins de fer hors série, $\mathrm{n}^{\circ}$ 4, Paris, AHICF, 1996.

KLARSFELD (Serge), " L'acheminement des Juifs de province vers Drancy et les déportations ", in AHICF, Une entreprise publique dans la guerre. La SNCF, 1939-1945, Paris, PUF, 2001, p. 143-158.

LAVIGNE (Raymond), Saint-Pierre-des-Corps ou la clarté républicaine, Paris, Messidor, 1988, $160 \mathrm{p}$.

LE MANER (Yves), Le Train de Loos, le grand drame de la déportation dans le Nord - Pas-de-Calais, chez l'auteur, 2003, 263 p.

DELATTRE (Maxime), "L'épuration administrative à la SNCF à la libération », mémoire de maitrise d'histoire, sous la dir. de Nadine Vivier, université du Maine, septembre 2002, 153 p. [Déposé à l'AHICF.]

MARGAIRAZ (Michel), «La SNCF, l'État français, l'occupant et les livraisons de matériel : la collaboration ferroviaire d'État en perspective », in AHICF, Une entreprise publique dans la guerre. La SNCF, 1939-1945, Paris, PUF, 2001, p. 71-82.

MATTASSOLIO (Régine), " Les cheminots à Dôle et Besançon durant la Deuxième Guerre mondiale », mémoire de maîtrise d'histoire, université de Bourgogne, 1990.

MENCHERINI (Robert) ; DOMENICHINO (Jean) ; LAMOUREUX (David), Cheminots en Provence. Des voix de la mémoire aux voies de l'avenir (18302001), Paris, Éditions La Vie du rail, 2001, 247 p.

Chapitre IV, p. 114-139 : "Les cheminots, des années noires à la Libération. »

PLANCKE (René-Charles), Le Chemin de fer de Seine-et-Marne, tome 1, De la vapeur au TGV, Dammarie-les-Lys, Amatteis, 1991, 512 p.

RIBEILL (Georges), « Le personnel de la SNCF (1937-1981). Contraintes économiques, issues techniques, mutations professionnelles et évolutions sociales. Les cours successifs d'une entreprise publique », Paris, Développement et aménagement, 1982, ronéo., xiii, 633 p. 
RIBEILL (Georges), Les Cheminots, Paris, Éditions La Découverte, 1984, $127 \mathrm{p}$.

RIBEILL (Georges), "La société cheminote: quelques pistes pour la recherche historique », Revue d'histoire des chemins de fer, $\mathrm{n}^{\circ} 1$ (automne 1989), p. 45-67.

RIBEILL (Georges), "Cultures d'entreprises : le cas des cheminots, des Compagnies à la SNCF », Ethnologie de la France, cahier 4, numéro spécial Cultures du travail. Identités et savoirs industriels dans la France contemporaine, Paris, Éditions de la Maison des sciences de l'homme, 1989, p. 251-265.

RIBEILL (Georges), "Les cheminots : esquisse d'un bilan social », in Denis Peschanski et Jean-Louis Robert (dir.), Les Ouvriers en France pendant la Seconde Guerre mondiale, Actes du colloque des 22-24 octobre 1992, Paris, Centre d'histoire des mouvements sociaux et du syndicalisme / université Paris I / IHTP-CNRS, 1992, p. 141-154.

RIBEILL (Georges), "Les métamorphoses d'une corporation », in Maurice Lemoine, Georges Ribeill et Anna Malan, Les Cheminots. Que reste-t-il de la grande famille?, Paris, Syros, 1993, p. 123-205.

RIBEILL (Georges), "Les militants cheminots : quelques approches à partir du Maitron », in Michel Dreyfus, Claude Pennetier et Nathalie Viet-Depaule (dir.), La Part des militants, Paris, Les Éditions de l'atelier - Les Éditions ouvrières, 1996, p. 133-144.

RIEDWEG (Eugène), " La SNCF en Alsace et Moselle de 1939 à 1945 : le rôle des cheminots ", in AHICF, Une entreprise publique dans la guerre. La SNCF, 1939-1945, Paris, PUF, 2001, p. 205-211.

SCHONTZ (André), Le Chemin de fer et la gare de Metz, Metz, Serpenoise, 1990, 224 p.

TESSIER DU CROS (Henri), Louis Armand. Visionnaire de la modernité, Paris, Odile Jacob, 1987, 500 p.

THOMAS (Pierre), Septembre 1939 - mai 1940. Des trains contre les panzers, s.l., La Voix du Nord, 1999, 238 p.

Sur la SNCF Région nord pendant les combats.

VIRARD-PETITJEAN (Elisabeth), "Aspects du monde de la SNCF dans la région lyonnaise entre 1940 et 1944 », mémoire de maîtrise d'histoire, sous la dir. de Gilbert Garrier, université Lumière - Lyon II, 1979, 123 p.

WOLKOWITCH (Maurice), «Le réseau ferré français du débarquement du 6 juin 1944 à la capitulation de l'Allemagne le 8 mai 1945 », Revue d'histoire des chemins de fer, $\mathrm{n}^{\circ} 15$ (automne 1996), p. 225-250. 


\section{I'Engagement résistant}

3.1. Généralités sur l'engagement résistant (ouvrages et articles)

* Différenciation entre "résistance " et autres formes d'opposition à l'occupant (légales, par exemple)

LABORIE (Pierre), "L'idée de Résistance, entre définition et sens : retour sur un questionnement », in « La Résistance et les Français. Nouvelles approches », Les Cahiers de l'IHTP, n 37 (décembre 1997), p. 15-27.

MARCOT (François), «Qu'est-ce qu’un patron résistant? » in Olivier Dard, Jean-Claude Daumas et François Marcot (dir.), L'Occupation, l'État français et les entreprises, Actes du colloque organisé par l'université de Franche-Comté et le Musée de la Résistance et de la Déportation de Besançon, 24-26 mars 1999, Paris, ADHE, 2000, p. 277-292.

SEMELIN (Jacques), "Jalons pour une histoire de la France résistante » in Laurent Douzou, Robert Franck, Denis Peschanski et Dominique Veillon (dir.), La Résistance et les Français : villes, centres et logiques de décision, Actes du colloque international de Cachan, 16-18 novembre 1995, Paris, IHTP, 1996, p. 459-468.

SEMELIN (Jacques), Sans armes face à Hitler. La résistance civile en Europe, 1939-1945, Paris, Payot, 1998 (1 ${ }^{\text {re }}$ édition 1989), 274 p.

\section{* Facteurs explicatifs de l'engagement et sa chronologie}

DOUZOU (Laurent), «L'entrée en résistance », in Antoine Prost (dir.), La Résistance, une histoire sociale, Paris, Les Éditions de l'atelier/Les Éditions ouvrières, 1997, p. 9-20.

MARCOT (François), « Pour une sociologie de la Résistance : intentionnalité et fonctionnalité », Antoine Prost (dir.), La Résistance, une histoire sociale, Paris, Les Éditions de l'atelier/Les Editions ouvrières, 1997, p. 21-41.

\section{* Motivations et valeurs de référence des résistants}

MARCOT (François), « Réflexions sur les valeurs de la Résistance » in JeanMarie Guillon et Pierre Laborie (dir.), Mémoire et histoire : la Résistance, Toulouse, Privat, 1995, p. 81-90.

SAINCLIVIER (Jacqueline) ; VEILLON (Dominique), " Sens et formes de la Résistance française », in «La Résistance et les Français. Nouvelles approches », Les Cahiers de l'IHTP, n 37 (décembre 1997), p. 93-108.

WIEVIORKA (Olivier), «Pour une lecture critique de l'engagement résistant ; l'exemple de Défense de la France », in Jean-Marie Guillon et Pierre Laborie (dir.), Mémoire et histoire : la Résistance, Toulouse, Privat, 1995, p. 91-98. 


\subsection{Sur l'engagement des cheminots}

CHEVANDIER (Christian), «La résistance des cheminots : le primat de la fonctionnalité plus qu'une réelle spécificité » in Antoine Prost (dir.), La Résistance, une histoire sociale, Paris, Les Éditions de l'atelier/Les Éditions ouvrières, 1997, p. 147-158.

MOISSONNIER (Maurice), "Les ouvriers du Rhône, de la France de Daladier à celle de Pétain : réactions aux conditions matérielles et morales ", in Denis Peschanski et Jean-Louis Robert (dir.), Les Ouvriers en France pendant la Seconde Guerre mondiale, Actes du colloque des 22-24 octobre 1992, Paris, Centre d'histoire des mouvements sociaux et du syndicalisme / université Paris I / IHTP-CNRS, 1992, p. 103-117.

p. 113 et 115, sur l'attitude des cheminots d'Oullins.

RIBEILL (Georges), "Les cheminots : esquisse d'un bilan social », in Denis Peschanski et Jean-Louis Robert (dir.), Les Ouvriers en France pendant la Seconde Guerre mondiale, Actes du colloque des 22-24 octobre 1992, Paris, Centre d'histoire des mouvements sociaux et du syndicalisme / université Paris I / IHTP-CNRS, 1992, p. 141-154.

Voir la fin de l'article.

RIBEILL (Georges), «Les cheminots face à la lutte armée. Les différenciations sociologiques de l'engagement résistant », in François Marcot (dir.), La Résistance et les Français : Lutte armée et maquis, Colloque de Besançon, Annales littéraires de l'université de Franche-Comté, 1996, p. 71-81.

Comparaison avec d'autres corporations

ROUQUET (François), «Solidarités, engagements et 'ordres de Résistance’ aux PTT », in Jean-Marie Guillon et Pierre Laborie (dir.), Mémoire et histoire : la Résistance, Toulouse, Privat, 1995, p. 289-296.

KITSON (Simon), "L'évolution de la Résistance dans la police marseillaise », in Jean-Marie Guillon et Robert Mencherini (dir.), La Résistance et les Européens du Sud, Paris, L'Harmattan, 1999, p. 257-270. 


\section{Les formes de résistance et les types d'organisation}

4.1. Problématique générale sur les formes d'action et les types d'organisations résistantes

* Relations entre formes de résistance et possibilités d'organisation, la surévaluation a posteriori de l'organisation planifiée sur l'adaptation, l'explication des hiérarchies au sein des groupes

DOUZOU (Laurent), «Organisations et modes de fonctionnement de la Résistance », in « La Résistance et les Français. Nouvelles approches », Les Cahiers de l'IHTP, nº 37 (décembre 1997), p. 109-127.

MARCOT (François), «Dans quelle mesure les villes exercent-elles un pouvoir de commandement et d'orientation sur la Résistance dans son ensemble? ", in La Résistance et les Français : villes, centres et logiques de décision, Actes du colloque international de Cachan, 16-18 novembre 1995, Paris, IHTP, 1996, p. 215-228.

* Poids des cultures propres aux groupes sociaux (politique, corporatiste, syndicale), relation entre la résistance organisée et son environnement

MARCOT (François), «La Résistance dans ses lieux et milieux : des relations d'interdépendance », in « La Résistance et les Français. Nouvelles approches », Les Cahiers de l'IHTP, n 37 (décembre 1997), p. 129146.

VIRIEUX (Daniel), "Résistance - Professions. Un rapport sans histoire(s)? ", in Antoine Prost (dir.), La Résistance, une histoire sociale, Paris, Les Éditions de l'atelier/Les Éditions ouvrières, 1997, p. 113146.

\section{* Les stratégies, notamment en matière de lutte armée}

MARCOT (François) (dir.), La Résistance et les Français. Lutte armée et maquis, Actes du colloque international de Besançon, 15-17 juin 1995, Annales littéraires de l'université de Franche-Comté, 1996, 549 p.

\section{* Sur l'approche sociologique}

KEDWARD (Harry Roderick), Naissance de la Résistance dans la France de Vichy. Idées et motivations, 1940-1942, Seyssel, Champ Vallon, 1989, 351 p. (Vichy France and the Resistance: culture and ideology, Londres / Sydney, Croom Helm, cop., 1985.)

MARCOT (François), « Pour une sociologie de la résistance : intentionnalité et fonctionnalité », in Antoine Prost (dir.), La Résistance, une histoire sociale, Paris, Les Éditions de l'atelier/Les Éditions ouvrières, 1997, p. 21-41. 
* Sociologie de la Résistance : études départementales et régionales

BOIVIN (Michel); QUELLIEN (Jean), «La Résistance en BasseNormandie : Définition et sociologie », in Jacqueline Sainclivier et Christian Bougeard (dir.), La Résistance et les Français. Enjeux stratégiques et enironnement social, Actes du colloque international de Rennes, 2930 septembre $-1^{\text {er }}$ octobre 1994, Rennes, Presses universitaires de Rennes, p. 163-173.

GABERT (Michèle), Entrés en Résistance. Isère. Des hommes et des femmes dans la Résistance, Grenoble, Presses universitaires de Grenoble, 2000, 350 p.

MATHIEU (Eric), « Sociologie de la Résistance dans le Puy-de-Dôme », in André Gueslin (dir.), De Vichy au Mont Mouchet. L'Auvergne en guerre, 1939-1945, Institut d'études du Massif central, 1991, p. 121-146.

SAINCLIVIER (Jacqueline), « Sociologie de la résistance : quelques aspects méthodologiques et leur application en Ille-et-Vilaine », Revue d'histoire de la Deuxième Guerre mondiale, n 117 (janvier 1980), p. 33-74.

SAINCLIVIER (Jacqueline), La Résistance en Ille-et-Vilaine, 1940-1944, Rennes, PUR, 1993, 332 p.

\section{* Sur l'engagement des femmes, des familles}

ANDRIEU (Claire), "Les résistantes, perspectives de recherche », in Antoine Prost (dir.), La Résistance, une histoire sociale, Paris, Les Éditions de l'atelier/Les Éditions ouvrières, 1997, p. 69-96.

DOUZOU (Laurent), « La Résistance, une affaire d’hommes? », Les Cahiers de l'IHTP, n 31 (octobre 1995), p. 11-24.

DOUZOU (Laurent), "Les résistantes, point de l'historiographie », in Mechtild Gilzmer, Christine Levisse-Touzé et Stefan Martens (dir.), Les Femmes dans la Résistance en France, Actes du colloque international de Berlin, 8-10 octobre 2001, Paris, Tallandier, 2003, p. 31-49.

Sur les cheminots

RIBEILL (Georges), « Les cheminots face à la lutte armée. Les différenciations sociologiques de l'engagement résistant », in La Résistance et les Français : Lutte armée et maquis, Colloque de Besançon, Annales littéraires de l'université de Franche-Comté, 1996, p. 71-81.

\subsection{Formes de résistance des cheminots}

* Ouvrages généraux/sources sur la résistance cheminote et les cheminots résistants

L'Appel du cheminot ancien combattant, bulletin de l'Association nationale des cheminots anciens combattants, résistants, prisonniers et victimes de guerre. 
Union nationale des anciens combattants et victimes de guerre des chemins de fer, 1974-...

"Aux cheminots morts pour la France ", La Vie du rail, nº spécial, 25 août 1964, 84 p.

Résistance-Fer, Bataille du Rail, 44 p. (non daté).

ARMAND (Louis), Propos ferroviaires, Paris, Fayard, 1971, chap. 3 et 4, p. 75-114.

CHOURY (Maurice), Les Cheminots dans la bataille du rail, Paris, Perrin, 1970, $371 \mathrm{p}$.

DAVID (François), «Léon Bronchart (1896-1986) ou "les vertus du peuple" ", in Visages de la Résistance en pays de Brive, Brive, Les Trois Epis, 1998, p. 168-176.

MÉCHIN (A.), «Louis Armand et la Résistance », Le Déporté. Organe mensuel de l'UNADIF, $\mathrm{n}^{\circ} 277$ (septembre 1971).

DURAND (Paul), La SNCF pendant la guerre, sa résistance à l'occupant, Paris, PUF, coll. «Esprit de la Résistance », 1968, 666 p.

Ensuite, pour chaque type d'actions de résistance, on rappelle en premier les ouvrages de référence, puis les ouvrages spécifiques éventuels concernant les seuls cheminots.

\section{2.a) Aide aux réfractaires, hébergement, faux papiers}

\section{* Sur le STO}

ÉVRARD (Jacques), La Déportation des travailleurs français dans le III' Reich, Paris, Fayard, 1971, 461 p.

GARNIER (Bernard); QUELLIEN (Jean) (dir.), La Main-d'cuure française exploitée par le III Reich, Actes du colloque international, 13-15 décembre 2001, Caen, Centre de recherche d'histoire quantitative, 2003, 704 p.

4.2.b) Évasions et passages, transports de courrier et de matériel Association française des déportés évadés des trains de déportation, Mémorial, s.l., AFDETD, 1999, non paginé.

ALARY (Éric), La Ligne de démarcation 1940-1944, Paris, Perrin, 2003, $432 \mathrm{p}$.

Notamment p. 119-123 sur la complicité des cheminots dans les passages clandestins de la ligne, sur les formes que peut prendre cette complicité.

BONNOT (Thierry), «Les passeurs clandestins de la ligne de démarcation en Saône-et-Loire », mémoire de DEA d'histoire, université de Bourgogne, 1993. 
BONNOT (Thierry), La Ligne de démarcation en Saône-et-Loire pendant la Seconde Guerre mondiale, Génelard, Le Caractère en marche, 1994, $179 \mathrm{p}$.

CHIROL (Jean-Marie), 5 juin 1944, entre Chalons et Vitry-le-François, sur les chemins de l'enfer. 45 déportés s'évadent d'un train partant pour Neuengamme, 1996, $124 \mathrm{p}$.

LEMOINE (Henri), Les Évasions des convois de déportation, s.l., s.e., 1993, $24 \mathrm{p}$.

p. 15 : sur l'aide apportée par les cheminots.

LUTAUD (Laurent) ; DI SCALA (Patricia), Les Naufragés et les rescapés du "Train fantôme », Paris, L'Harmattan, 2003, 249 p.

PROVOST (Sylvie), "Le train pour l'évasion de Paul Langevin (2 au 5 mai 1944)", in AHICF, Une entreprise publique dans la guerre. La SNCF, 1939-1945, Paris, PUF, 2001, p. 281-291.

\section{2.c) Grèves et manifestations}

TARTAKOWSKY (Danielle), « Ouvriers et manifestations de rue : 19401944. Des manifestations ouvrières ? » in Denis Peschanski et Jean-Louis Robert (dir.), Les Ourriers en France pendant la Seconde Guerre mondiale, Actes du colloque des 22-24 octobre 1992, Paris, Centre d'histoire des mouvements sociaux et du syndicalisme / université Paris I / IHTP-CNRS, 1992, p. 419-427.

Mention des cheminots pour des manifestations de 1943.

TARTAKOWSKY (Danielle), Les Manifestations de rue en France, 1918-1968, Paris, Publications de la Sorbonne, 1997, 869 p.

Sur les cheminots

1944-1994.50 anniversaire de la grève insurrectionnelle des cheminots, Fédération CGT des cheminots, mai 1994.

SNCF CCR Paris Nord, "10 août 1944. Cinquantenaire de la Grève Insurrectionnelle. Les cheminots de la Région de Paris-Nord témoignent », Voies libres, numéro supplémentaire, 10 août 1994, 22 p.

CHEVANDIER (Christian), «Oullins 1942 : les cheminots en grève contre la collaboration ", Correspondances, $n^{\circ} 11$ (février 2004), p. 42-47.

CHEVANDIER (Christian), Cheminots en grève ou la construction d'une identité (1848-2001), Paris, Maisonneuve \& Larose, 2002, 399 p.

Notamment chap. 3 : «Les batailles du rail (1939-1944)», p. 155-218.

MOISSONNIER (Maurice), "13-17 octobre 1942. Il y a 50 ans, à Lyon, les premières grandes grèves en zone non occupée. La réponse des cheminots et des métallos à Laval et Sauckel », Documents de l'Institut CGT d'histoire sociale Rhône-Alpes, numéro spécial, octobre 1992, p. 7-18. 


\section{2.d) Maquis}

KEDWARD (Harry Roderick), À la recherche du Maquis. La Résistance dans la France du Sud, 1942-1944, Paris, Les Éditions du Cerf, 1999, 473 p.

MARCOT (François) (dir.), La Résistance et les Français. Lutte armée et maquis, Actes du colloque international de Besançon, 15-17 juin 1995, Annales littéraires de l'université de Franche-Comté, Besançon, 1996, 549 p.

4.2.e) Presse et propagande en général (transport, diffusion notamment; presse dédiée aux cheminots : rédaction, contenu)

Presse clandestine (La) 1940-1944, colloque d'Avignon, 20-21 juin 1985, Conseil général de Vaucluse, 1986.

CRÉMIEUX-BRILHAC (Jean-Louis) (dir.), Les Voix de la Liberté- Ici Londres (1940-1944), Paris, La Documentation Française, 1975, 5 volumes.

MARCOT (François), La Franche-Comté sous l'Occupation, tome 2, Les Voix de la Résistance. Tracts et journaux clandestins francs-comtois, Besançon, Cêtre, 1989, 367 p.

PARDIEU (Laurent), "Un journal clandestin dans la zone interdite : "Lorraine » - 1942-1944» mémoire de maitrise d'histoire, sous la dir. de François Roth, université Nancy II, 1991.

ROUX-FOUILLET (Paul) ; ROUX-FOUILLET (Renée), Catalogue des périodiques clandestins conservés à la Bibliothèque Nationale (1939-1945), Paris, Bibliothèque nationale, 1954, 282 p.

\section{2.f) Renseignement}

PASSY (Colonel), Mémoires du chef des services secrets de la France Libre, Paris, Odile Jacob, 2000, 801 p.

\section{2.g) Sabotages et attentats, freinage}

MARCOT (François) (dir.), La Résistance et les Français. Lutte armée et maquis, Actes du colloque international de Besançon, $15-17$ juin 1995, Annales littéraires de l'université de Franche-Comté, Besançon, 1996, 549 p.

$\underline{\text { Sur les cheminots }}$

Résistance FFI, Fédération des amicales des Forces Françaises de l'Intérieur du Doubs, Jura nord, Territoire de Belfort, 1976-...

Nombreux articles sur les sabotages.

BESSON (André), « Sabotage à Mouchard », in Une poignée de braves. Épisodes de la Résistance franc-comtoise, 1949-1944, Poligny, Les Nouvelles Éditions jurassiennes, 1965, p. 141-150.

MARCOT (François), "Les sabotages ferroviaires dans le Doubs et le Jura nord sous l'Occupation », mémoire de maitrise d'histoire, université de Paris I, 1971. 
PENAUD (Guy), Les Milliards du train de Nemirc, Périgueux, FANLAC, 2001, $156 \mathrm{p}$.

QUELLIEN (Jean), Résistance et sabotages en Normandie. Le MaastrichtCherbourg déraille à Airan, Condé-sur-Noireau, Éditions Charles Corlet, 1992, 141 p., (nouvelle éd., 2004).

ROMANS PETIT (Henri), 52 locomotives, Saint-Étienne, Stéfa, 1946, 31 p. Récit romancé du sabotage d’Ambérieu, 6 juin 1944.

VADON (Jacques), Contribution à l'histoire de la Résistance dans les Ardennes (juin 1940-septembre 1944). Chronologie des principaux événements et liste des sabotages ferroviaires, Charleville-Mézières, Centre départemental de documentation pédagogique, 1969, $112 \mathrm{p}$.

\section{2.h) NAP}

BARUCH (Marc Olivier), Servir l'État français. L'administration en France de 1940 à 1944, Paris, Fayard, 1997, 737 p.

BOURDET (Claude), L'Aventure incertaine. De la Résistance à la Restauration, Paris, Stock, 1975, 479 p.

GRANET (Marie) ; MICHEL (Henri), Combat. Histoire d'un mouvement de Résistance de juillet 1940 à juillet 1943, Paris, PUF, 1957, 330 p.

VISTEL (Alban), La Nuit sans ombre. Histoire des mouvements unis de la Résistance, leur rôle dans la libération, Paris, Fayard, 1970, 640 p.

Sur les cheminots

Association des amis de Louis Armand, [Henri Malcor], Louis Armand. 40 ans au service des hommes, Paris, Charles Lavauzelle, 1986, $172 \mathrm{p}$. p. 25-46 : chapitre III : «La guerre », notamment sur NAP-Fer, Plan vert et l'arrestation en juin 1944.

DURAND (Paul), La SNCF pendant la guerre, sa résistance à l'occupant, Paris, PUF, coll. « Esprit de la Résistance », 1968, 666 p.

HEILBRONN (Max); VARIN (Jacques), Galeries Lafayette, Buchenwald, Galeries Lafayette, Paris, Economica, 1989, 177 p.

TESSIER DU CROS (Henri), Louis Armand. Visionnaire de la modernité, Paris, Odile Jacob, 1987, 500 p.

p. 129-170 : «Le compagnon de la Libération », quelques pages sur le NAP-Fer, sur l'arrestation de juin 1944. 


\subsection{Types d'organisation}

\section{3.a) Partis et syndicats}

Mouvement syndical dans la Résistance (Le), s.l., La Courtille, 1975, 222 p.

AZÉMA (Jean-Pierre) ; PROST (Antoine) ; RIOUX (Jean-Pierre) (dir.), Le Parti communiste français des années sombres: 1938-1941, Actes du colloque des 14 et 15 octobre 1983, Paris, Le Seuil, 1986, 321 p.

COURTOIS (Stéphane), Le PCF dans la guerre : de Gaulle, la Résistance, Staline..., Paris, Ramsay, 1980, 585 p.

LE CROM (Jean-Pierre), Syndicats nous voilà! Vichy et le corporatisme, Paris, Les Éditions de l'atelier / Les Éditions ouvrières, 1995, 410 p.

LE CROM (Jean-Pierre), «Syndicalisme et résistance », in La Résistance et les Français : villes, centres et logiques de décision, Actes du colloque international, Cachan, 16-18 novembre 1995, p. 397-413.

RIOUX (Jean-Pierre) ; PROST (Antoine); AZÉMA (Jean-Pierre) (dir.), Les Communistes français de Munich à Chatteaubriant (1938-1941), Paris, Presses de la Fondation nationale des sciences politiques, 1987, 439 p.

Nombreuses mentions du rôle essentiel des cheminots dans la réorganisation du PCF après juin 1940, avec exemples régionaux.

SADOUN (Marc), Les socialistes sous l'occupation: Résistance et collaboration, Paris, Presses de la Fondation nationale des sciences politiques, 1982, $323 \mathrm{p}$.

TOLLET (André), La Classe ouvrière dans la résistance, Paris, Messidor/ Éditions sociales, 1983, 296 p.

Sur les cheminots

BROUDER (Annie), «La fédération CGT des cheminots de novembre 1938 à février 1943 : de la scission à la réunification », mémoire de maitrise d'histoire, sous la direction de J. Droz et J. Girault., Sorbonne, 1970, $183 \mathrm{p}$.

CGT - Secteur fédéral des cheminots, région de Strasbourg, Ily a 50 ans, Georges Wodli, Montreuil, CGT, 1993, 12 p.

CHAUMEL (Guy), Histoire des cheminots et de leurs syndicats, Paris, Rivière, $1948,200 \mathrm{p}$.

S'arrête avant la guerre, quelques pages sur la période 1939-1945 en conclusion.

DUPUY (Jean-Marie), « Les communistes alsaciens » in Saisons d'Alsace, $\mathrm{n}^{\circ} 114$ (hiver 1991-1992), p. 257-266.

Sur Wodli.

Fédération des cheminots CGT, Il y a 50 ans Pierre Sémard. Les résistants mais aussi les idées toujours novatrices, [1992], 13 p.

GACON (Jean), et alii, Les Batailles du rail, Paris, Messidor, 1986, 221 p. 
JACQUET (Joseph), et alii, Les Cheminots dans l'histoire sociale de la France, Paris, Éditions sociales, 1967, 318 p.

RIBEILL (Georges), " Les chantiers de la collaboration sociale des Fédérations légales des cheminots (1939-1944)», Le Mouvement social, $\mathrm{n}^{\circ} 158$ (janvier-mars 1992), p. 87-116.

VINCENT (Pierre), «Le syndicalisme sous Vichy : le cas des cheminots », Les Cahiers de l'Institut, $\mathrm{n}^{\circ} 28$ (1 $1^{\mathrm{er}}$ trim. 2006), p. 21-27.

4.3.b) Mouvements et groupes locaux

AGLAN (Alya), La Résistance sacrifiée. Le mouvement "Libération-Nord », Paris, Flammarion, 1999, 456 p.

BÉDARIDA (Renée), Les Armes de l'esprit. Témoignage chrétien (1941-1944), Paris, Les Éditions ouvrières, 1977, 378 p.

BRUNEAU (Françoise), Essai d'historique du mouvement né autour du journal clandestin « Résistance », Paris, SEDES, 1951, 213 p.

CALMETTE (Arthur), L'OCM. Histoire d'un mowiement de résistance de 1940 à 1946, Paris, PUF, 1961, 228 p.

GRANET (Marie), " Défense de la France ». Histoire d'un Mouvement de Résistance (1940-1944), Paris, PUF, 1960, 302 p.

GRANET (Marie), Ceux de la Résistance, Paris, Éditions de minuit, 1964, $375 \mathrm{p}$.

GRANET (Marie) ; MICHEL (Henri), Combat. Histoire d'un mouvement de Résistance de juillet 1940 à juillet 1943, Paris, PUF, 1957, 330 p.

HOBAM (Nicolas), Quatre années de lutte clandestine en Lorraine. (Historique $d u$ "Mouvement Lorraine »), s.l., Les Éditions du bastion, 2002 ( $1^{\text {re }}$ édition 1946), $496 \mathrm{p}$.

PIGNOT (Jean-Pierre), " Aspects de la Résistance à Toulouse et dans sa région : "Libérer et Fédérer" », mémoire de maîtrise d'histoire, sous la dir. de Rolande Trempé, université Toulouse II - Le Mirail, 1976, $157 \mathrm{p}$.

PLÉ (Nicole), «Le mouvement de Résistance Libérer et Fédérer à Toulouse ", mémoire de maîtrise d'histoire, sous la direction de Robert Bonnaud, université Paris VII, 1987.

QUELLIEN (Jean); VICO (Jacques), Massacres naris en Normandie. Les fusillés de la prison de Caen, Condé-sur-Noireau, Éditions Charles Corlet, 1994, 234 p.

Cf. notamment chapitre IX sur « Le groupe du Front national des cheminots de la gare de Caen ».

VEILLON (Dominique), Le Franc-Tireur. Un journal clandestin, un mouvement de Résistance, 1940-1944, Paris, Flammarion, 1977, 428 p. 
VIRIEUX (Daniel), «Le Front national de lutte pour la liberté et l'indépendance de la France. Un mouvement de Résistance - Période clandestine (mai 1941 - août 1944) », thèse de doctorat d'histoire, sous la direction de $\mathrm{Cl}$. Willard, université Paris 8 Vincennes - SaintDenis, 1995, 5 volumes, 1824 p.

WETTERWALD (François), Vengeance, histoire d'un corps franc, Paris, Mouvement Vengeance, 1946, 302 p.

Voir notamment chap. $6:$ «Les Corps-Francs « Vengeance» SNCF », p. 50 sq., et chap. 19 : «Activités des Corps-Francs SNCF », p. 156 sq.

WIEVIORKA (Olivier), Une certaine idée de la France. Défense de la France, 1940-1949, Paris, Le Seuil, 1995, 496 p.

\section{3.c) Réseaux}

Mémorial du réseau Alliance

AGLAN (Alya), Mémoires résistantes. Histoire du réseau Jade-Fitroy 1940-1944, Paris, Les Éditions du Cerf, 1994, 344 p.

BAEHREL (Sylvaine), Alibi (1940-1944). Histoire d'un réseau de renseignement en France pendant la Deuxième Guerre mondiale, Paris, Jean-Michel Place, 2000, $214 \mathrm{p}$.

CUMONT (Jacques), Les Volontaires de Nenilly-sur-Marne du " groupe Hildevert " et le réseau «Spiritualist ». Le massacre d'Oissery, Le Mée-sur-Seine, Lys Éditions, 1991, 159 p.

DUCOUDRAY (Marie), Ceux de «Manipule». Un réseau de renseignements dans la Résistance en France, Paris, Tirésias, 2001, 277 p.

GRANET (Marie), Cohors-Asturies, histoire d'un réseau de Résistance, $1942-$ 1944, Bordeaux, Éditions des Cahiers de la Résistance, 1974, 117 p.

LECLÈRE (Françoise), « La composition d'un réseau « Zéro France » », Revue d'histoire de la Deuxième Guerre mondiale, nº 61 (janvier 1966), p. $75-86$.

Employés SNCF : profession la plus représentée dans ce réseau franco-belge d'évasions puis de renseignements notamment dans le secteur parisien.

LHEUREUX (Danièle), La Résistance «Action-Buckmaster ». Sylvestre-Farmer avec le capitaine "Michel 》, Roubaix, Geai Bleu éditions, 2001, 173 p. ; La Résistance "Action Buckmaster». Sylvestre Farmer sans le capitaine "Michel», Roubaix, Geai Bleu éditions, 2002, 259 p.

MEYSSONNIER (Jean-Philippe), « Le réseau Gallia, 1943-1944 », mémoire de DEA d'histoire, IEP de Paris, 1994, 208 p.

VEILLON (Dominique), "Les réseaux de Résistance », in Jean-Pierre Azéma et François Bédarida (dir.), La France des années noires. 1. De la défaite à Vichy, Paris, Points histoire, 2000, p. 407-439. 
4.3.d) Monographies régionales sur la résistance (organisations, formes d'action)

\section{* Ouvrages généraux (sélection)}

Association Résistance 60, La Résistance dans l'Oise, DVD-ROM édité par le CRDP d'Amiens et l'AERI, 2003.

BAUDOT (Marcel), Libération de la Normandie, Paris, Impr. Sully, 1974, $255 \mathrm{p}$.

BESSE (Jean-Pierre), L'Oise. Septembre 1940 - septembre 1944, auto-édition, 1994, 218 p.

CANAUD (Jacques), Les Maquis du Morvan (1943-1944). La vie dans les maquis, Château-Chinon, Académie du Morvan, 1981, 425 p.

CASTEL (Jean-Marie), Les Villeneuvois et les Villenemvoises sous l'Occupation, 1940-1944, Montgeron, Desbouis Gresil, 1990, 364 p.

CHAUMET (Michel) ; POUPLAIN (Jean-Marie), La Résistance en Deux-Sèrres (1940-1944), s.l., Geste Édition, 1994, 289 p.

CLESSE (Joël) ; ZAIDMAN (Sylvie), La Résistance en Seine-Saint-Denis, 1940 1944, Paris, Syros, 1994, 480 p.

COURVOISIER (André), Le Réseau Heckler : de Lyon à Londres, Paris, FranceEmpire, 1984, 299 p.

DEBON (André) ; PINSON (Louis), La Résistance dans le bocage normand, Paris, Tirésias, 1994, 348 p.

DEJONGHE (Étienne) ; LE MANER (Yves), Le Nord - Pas-de-Calais dans la main allemande, Lille, La Voix du Nord, 1999, 400 p.

DI CARLO (Jacqueline), La Guerre de 1939/1945 dans le canton de SaintRambert-en-Bugey. Épisodes.

DODIN (Robert), "Les F.F.I. en Lorraine », Revue d'histoire de la Deuxième Guerre mondiale, $\mathrm{n}^{\circ} 105$ (janvier 1977), p. 49-78.

DOUZOU (Laurent) ; VEILLON (Dominique), "La Résistance des mouvements ; ses débuts dans la région lyonnaise (1940-1942) », in Jean-Marie Guillon et Pierre Laborie (dir.), Mémoire et histoire : la Résistance, Toulouse, Privat, 1995, p. 149-159.

DUCROS (Louis-Frédéric), Montagnes ardéchoises dans la guerre. 1. Genèse du 17 juin 1940 au 11 novembre 1942, Valence, L.-F. Ducros, 1974, 214 p.

DUCROS (Louis-Frédéric), Montagnes ardéchoises dans la guerre, du 12 novembre 1942 au 5 juin 1944. 2. La lutte clandestine, Valence, L.-F. Ducros, 1977, 446 p.

DUCROS (Louis-Frédéric), Montagnes ardéchoises dans la guerre, du 6 juin 1944 au 7 septembre 1944.3. Combats pour la Libération, Valence, L.-F. Ducros, 1981, 418 p. 
DUVERLIE (Dominique), "Les Picards face à l'occupation allemande : le département de la Somme du 20 mai 1940 au 3 septembre 1944 », thèse de troisième cycle, sous la dir. d'Adeline Daumard, université d'Amiens, 1979, 2 volumes, 191 p. et 74 p.

ÉTIENNE (Jean-Louis) ; GÉRARD (Pierre), « Débuts et développement de la Résistance en Meurthe-et-Moselle(1940-1943) », Revue d'histoire de la Deuxième Guerre mondiale, n 105 (janvier 1977), p. 29-48.

GOUBET (Michel) ; DEBAUGES (Paul), Histoire de la Résistance en HauteGaronne, Mercuès, Éditions Milan, 1992, 250 p.

GUILLON (Jean-Marie), «La Résistance dans le Var. Essai d'histoire politique », thèse d'État, sous la dir. de Émile Témime, université de Provence, 1989, 3 volumes, 1199 p.

HENNEQUIN (Gilles), Résistance en Côte d'Or, tome 2, chez l'auteur, 1996, $217 \mathrm{p}$.

HOBAM (Nicolas), Quatre années de lutte clandestine en Lorraine, s.l., Les Éditions du bastion, 2002 (1 $1^{\text {re }}$ édition 1946), 496 p.

HUGUEN (Roger), Par les nuits les plus longues. Réseaux d'évasions d'aviateurs en Bretagne (1940-1944), Saint-Brieuc, Les Presses bretonnes, 1976, 508 p.

HUGUEN (Roger), Chantier d'évasions. Carantec (1940-1944). Réseau SibirilAlliance, Spézet, Coop. Breizh, 2005, 143 p.

JIMENEZ DE ABERAS TURI CORTA (Juan Carlos), En passant par la Bidassoa. Le résean Comète au Pays-Basque, 1941-1944, Biarritz, J\&D, 1996, $183 \mathrm{p}$.

LECLERC (Marcel), La Résistance dans la Manche. Réseaux et mouvements, juin 1940 - août 1944, Cherbourg, Éditions la dépêche, 1982, 290 p.

LE GRAND (Alain) ; THOMAS (Georges-Michel), 1939-1945. Finistère, Brest, Éditions de la cité, 1987, 414 p.

LEROUX (Roger), Le Morbihan en guerre, 1939-1945, Mayenne, Imprimerie de la manutention, 1986, 671 p.

LESAGE (René), «La Résistance en Artois occidental (juin 1940-mai 1944) », Revue du Nord, hors série, n 13, 1998, 336 p.

LOCHMANN (Xavier) ; TROGNEUX (Alain) ; NEUSCHWANDER (Isabelle), Un département dans la Guerre, 1939-1945, CRDP Amiens, Conseil général de la Somme, Textes et documents sur la Somme, 1997, 91 p.

MARCOT (François), La Franche-Comté sous l'Occupation, tome 1. La Résistance dans le Jura, Besançon, Cêtre, 1985, 332 p.

MARCOT (François), La Franche-Comté sous l'Occupation, tome 2. Les Voix de la Résistance, Besançon, Cêtre, 1989, 367 p. 
MARTRES (Eugène), L'Auvergne dans la tourmente, 1939-1945. L'État français. L'Occupation. La Résistance. La Libération, Clermont-Ferrand, Éditions De Borée, 2000, 504 p.

PANICACCI (Jean-Louis), Les Alpes-Maritimes de 1939 à 1945 : un département dans la tourmente, Nice, Serre, 1989, 398 p.

PANICACCI (Jean-Louis), La Résistance aquréenne, Nice, Serre, 1994, 259 p.

PERRAUD-CHARMANTIER (André), La Guerre en Bretagne. Récits et portraits, Nantes, Éditions Aux portes du large, 1947, 2 tomes, 197 et 277 p.

PLANCKE (René-Charles), La Seine-et-Marne 1939-1945, 4 tomes, Dammarie-les-Lys, Éditions Amatteis, 1984-1987.

QUELLIEN (Jean), Opinions et comportements politiques dans le Calvados sous l'occupation allemande (1940-1944), Caen, Presses universitaires de Caen, 2001, 511 p.

ROMANS-PETIT (Henri), Les Maquis de l'Ain, Paris, Hachette, 1974, 167 p.

RUBY (Marcel), La Résistance à Lyon, Lyon, L'Hermès, 1979, 2 tomes, $1054 \mathrm{p}$.

SAINCLIVIER (Jacqueline), " La Résistance en Ille-et-Vilaine (1940-1944) », thèse de $3^{\mathrm{e}}$ cycle, sous la dir. de M. Denis, université Rennes II, 1978, 2 volumes, 392 p. et 125 p.

SAINCLIVIER (Jacqueline), La Résistance en Ille-et-Vilaine, 1940-1944, Rennes, Presses universitaires de Rennes, 1993, 322 p.

SAINCLIVIER (Jacqueline), La Bretagne dans la guerre, 1939-1945, Rennes, Éditions Ouest-France, 1994, 218 p.

SAINCLIVIER (Jacqueline), "Les débuts de la Résistance en zone occupée », in Jean-Marie Guillon et Pierre Laborie (dir.), Mémoire et histoire : la Résistance, Toulouse, Privat, 1995, p. 161-170.

THOMAS (Georges-Michel); LE GRAND (Alain), Le Finistère dans la guerre : l'occupation, Brest, Éditions de la cité, 1980, 429 p.

THOMAS (Georges-Michel); LE GRAND (Alain), Le Finistère dans la guerre : la Libération, Brest, Éditions de la cité, 1981, 569 p.

VEYRET (Patrick), Histoire de la Résistance armée dans l'Ain, Châtillon-surChalaronne, La Taillanderie, 1999, 196 p.

Sur les cheminots

CHABOT (Commandant) (GIROUSSE Henri), Les Batailles d'Ambérieu et de l'Albarine, juin-juillet 1944, Bourg-en-Bresse, Association des anciens des maquis Ain et Haut-Jura, 1984, 44 p.

CUYNET (Jean), La Bataille du rail en Franche-Comté : cheminots et résistants, Châtillon-sur-Chalaronne, La Taillanderie, 1997, 320 p. 
DARAN (Alain), «Le nœud ferroviaire lyonnais pendant l'occupation allemande. De la collaboration à la Résistance », mémoire de maitrise d'histoire, université Lyon III, 1993, 242 p.

DARTOUT (Serge), « La Résistance ferroviaire en Limousin, 1943-1945 », mémoire de maittrise d'histoire, Limoges, 1975.

FORTHOFFER (Joël), «La résistance des cheminots en zone annexée », in Vandenbussche (Robert) (dir.), Les Services publics et la Résistance en zone interdite et en Belgique (1940-1944), Actes du colloque de Bondues, 30 janvier 2004, Centre de recherche sur l'histoire de l'Europe du NordOuest / CEGES / université Charles-de-Gaulle -Lille 3, 2005, p. 83-99.

GOUIFFÈS (Mélanie), «La résistance des cheminots en Gironde durant le Second conflit mondial », mémoire de maitrise d'histoire, sous la dir. de Christophe Bouneau, université Michel-de-Montaigne - Bordeaux III, 2001, 211 p. [Déposé à l'AHICF.]

IMMELÉ (Coralie), « La Résistance des cheminots à Lyon (1940-1944) », mémoire de maitrise d'histoire, sous la dir. de Laurent Douzou, université Lumière - Lyon II, 2001, 152 p. [Déposé à l'AHICF.]

IMMELÉ (Coralie), « La résistance des cheminots dans le Rhône (19401944) », mémoire de DEA d'histoire, sous la dir. de Laurent Douzou, université Lumière - Lyon II, 2002, 157 p. [Déposé à l'AHICF.]

OUTTERYCK (Pierre) (dir.), Mémoire cheminote en Nord - Pas-de-Calais : cheminots et chemins de fer du Nord (1938-1948), Actes du colloque organisé à Roubaix les 17 et 18 novembre 1995 par le Comité d'établissement régional de la SNCF, Paris, Tirésias, 1999, 166 p.

OUTTERICK (Pierre), "Les cheminots du Nord face à l'occupation hitlérienne ", in AHICF, Une entreprise publique dans la guerre. La SNCF, 1939-1945, Paris, PUF, 2001, p. 249-258.

PERRON (Fabrice), «Cheminots dans la Résistance en Côte d'Or pendant la Deuxième Guerre mondiale », mémoire de maitrise d'histoire, université de Bourgogne, 1991.

PORCHEROT (Bertrand), " Cheminots et Résistance en Saône-et-Loire, 1940-1944 », mémoire de DEA d'histoire, sous la dir. de Serge Wolikow, université de Bourgogne, 2001, 279 p.

PRIGENT (Alain), TILLY (Serge), « La bataille du rail dans les Côtes-duNord », Les cahiers de la Résistance populaire, n 8/9, octobre 2000, 175 p. Contient notamment des figures très claires sur les méthodes de sabotages des voies, de nombreux témoignages et documents sur les sabotages en Côtes-d'Armor, sur les groupes de résistance.

RICHE (Jean), "La bataille du rail en Franche-Comté », in La FrancheComté sous l'occupation allemande et sa libération, Lons-le-Saunier, Éditions Marque-Maillard, 1980, p. 117-125. 
VANSTEENSKISTE (Robert), Des cheminots de Lens, Avion, Méricourt dans la Résistance, Lens, IPC, 1994, 96 p.

VINCENT (Alain), La Bataille du rail à Laroche-Migennes. Des luttes ouvrières à la Résistance, Précy-sous Thil, Éditions de l'Armançon, 160 p., réed. 2002.

VINCENT (Alain), Les Blés rouges. De la Résistance à la Libération. La bataille du rail à Laroche-Migennes, Précy-sous-Thil, Éditions de l'Armançon, 1996, 156 p.

VINCENT (Alain), Du fer au flambeau. De la Libération à la reconstruction, Précy-sous-Thil, Éditions de l'Armançon, 1997, 154 p.

\section{La répression individuelle et collective (arrestations, procès, trahisons, exécutions, massacres, sanctions collectives)}

\section{* Logiques à l'ouvre chez l'occupant (notamment à l'égard des com- munistes, des Juifs)}

MEYER (Ahlrich), « Les débuts du 'cycle attentats-répression’ en automne 1941 », in La Résistance et les Français : villes, centres et logiques de décision, Actes du colloque international de Cachan, 16-18 novembre 1995, Paris, IHTP, 1996, p. 486-497.

MEYER (Ahlrich), L'Occupation allemande en France (1940-1944), Toulouse, Privat, 2002, 238 p.

SOLCHANY (Jean), " Le commandement militaire en France face au fait résistant : logiques d'identification et stratégies d'éradication », in $L a$ Résistance et les Français : villes, centres et logiques de décision, Actes du colloque international de Cachan, 16-18 novembre 1995, Paris, IHTP, 1996, p. 511-530.

Répression à l'égard de cheminots

Lettres de fusillés, Paris, Éditions sociales, 1970, 127 p.

En particulier lettres de Catelas et Semard.

Vie à en mourir (La). Lettres de fusillés 1941-1944, Lettres choisies et présentées par Guy Krivopissko, Paris, Tallandier, 2003, 367 p.

KLARSFELD (Serge), Le Livre des otages, Paris, Les Éditeurs réunis, 1979, 295 p.

KLARSFELD (Serge) ; TSEVERY (Léon), Les 1007 fusillés du Mont-Valérien parmi lesquels 174 juifs, Paris, Association Les fils et filles des déportés juifs de France, 1995, 100 p.

MOCQ (Jean-Marie), La 12.SS Panzer-Division massacre Ascq cité martyre, s.l., Éditions Heimdal, 1994, 197 p. 
QUELLIEN (Jean); VICO (Jacques), Massacres nazis en Normandie. Les fusillés de la prison de Caen, Condé-sur-Noireau, Éditions Charles Corlet, 1994, $234 \mathrm{p}$.

ROSSEL-KIRSCHEN (André), Le Procès de la Maison de la Chimie (7 au 14 avril 1942). Contribution à l'histoire des débuts de la Résistance armée en France, Paris, L'Harmattan, 2002, 196 p.

Portrait de Louis Coquillet, cheminot breton, un des accusés du procès, fusillé au Mont-Valérien.

\section{La libération}

* Plans de sabotages et de guérilla, insurrection nationale; logiques des Alliés, du CFLN, des résistants

BUTON (Philippe), Les Lendemains qui déchantent. Le Parti communiste français à la Libération, Paris, Presses de la Fondation nationale des sciences politiques, 1993, $352 \mathrm{p}$.

CRÉMIEUX-BRILHAC (Jean-Louis), La France Libre. De l'appel du 18 Juin à la Libération, Paris, Gallimard, 1996, 969 p.

Notamment le chapitre 33, p. 772-795 : «L'insurrection nationale aura-t-elle lieu ?»

DELMAS (Colonel Jean), "Conceptions et préparation de l'insurrection nationale », in Actes du colloque La Libération de la France, Paris, 2831 octobre 1974, CNRS, 1976, p. 433-460.

FRANCK (Robert), "Identités résistantes et logiques alliées », in « La Résistance et les Français. Nouvelles approches », Les Cahiers de l'IHTP, n 37 (décembre 1997), p. 73-91.

MENCHERINI (Robert), Forces sociales, pouvoirs et société à Marseille, mémoire pour l'habilitation, université d'Aix-en-Provence, 1994.

MENCHERINI (Robert), "La ville comme élément de la stratégie », in La Résistance et les Français : villes, centres et logiques de décision, Actes du colloque international de Cachan, 16-18 novembre 1995, Paris, IHTP, 1996, p. 229-240.

\section{* L'insurrection parisienne}

Paris. Les heures glorieuses, août 1944. Le C.P.L. prépare et dirige l'insurrection, Montrouge, Imprimerie Draeger Frères, 1945, 105 p.

DANSETTE (Adrien), Histoire de la libération de Paris, Paris, Perrin, 1994, $483 \mathrm{p}$.

WIEVIORKA (Olivier), "La Résistance intérieure et la libération de Paris », in Christine Levisse-Touzé (dir.), Paris 1944. Les enjeux de la Libération, Paris, Albin Michel, 1994, p. 137-151. 
Sur la SNCF et les cheminots

WOLKOWITCH (Maurice), «Le réseau ferré français du débarquement du 6 juin 1944 à la capitulation de l'Allemagne le 8 mai 1945 », Revue d'histoire des chemins de fer, $\mathrm{n}^{\circ} 15$ (automne 1996), p. 225-250.

\section{La mémoire}

\section{* Sur les phénomènes de construction de la mémoire}

DOUZOU (Laurent), "La constitution du mythe de la Résistance », in Ch. Franck (dir.), La France de 1945. Résistances. Retours. Renaissances, Actes du colloque de Caen (17-19 mai 1995), Caen, Presses universitaires de Caen, 1996, p. 73-83.

DOUZOU (Laurent), « La mémoire de la Résistance dans la société française d'après-guerre », in Stanley Hoffman (dir.), Les courants politiques et la Résistance : continuités ou ruptures, Actes du colloque international d'Eschsur-Alzette, avril 2002, Luxembourg, Archives nationales, 2003, p. 490-503.

Institut d'histoire du temps présent, La Mémoire des Français. Quarante ans de commémoration de la Seconde Guerre mondiale, Paris, Éditions du CNRS, 1986, 400 p.

LABORIE (Pierre), "Sur les représentations collectives de la Résistance dans l'après-libération et les usages de la mémoire » in José Gotovitch et Robert Franck (dir.), La Résistance et les Européens du Nord, Actes du colloque de Bruxelles, 23-25 novembre 1994, Centre d'études et de recherches historiques de la Seconde Gerre mondiale / IHTP, 1994 et 1996, 460 p. et 158 p., tome 1, p. 419-423, ainsi que son intervention p. 144 dans le tome 2.

LABORIE (Pierre), « Opinions et représentations : la Libération et la construction de l'image de la Résistance ", in Les Français des années troubles. De la guerre d'Espagne à la Libération, Paris, Points histoire, 2003, p. 245267.

LABORIE (Pierre), «Honneur inventé ou invention du futur? Mémoire et appropriation de la Résistance à la Libération », in Les Français des années troubles. De la guerre d'Espagne à la Libération, Paris, Points histoire, 2003, p. 269-282.

ROUSSO (Henry), Le Syndrome de Vichy de 1944 à nos jours, Paris, Le Seuil, Points histoire, 1990 ( $1^{\text {re }}$ édition 1987), 416 p.

ROUSSO (Henry), Vichy. L'événement, la mémoire, l'histoire, Paris, Gallimard, Folio histoire, 2001, 748 p. 


\section{* Sur les " lieux de mémoire"}

Commission départementale de l'information historique pour la Paix, Les Lieux de mémoire de la Deuxième Guerre mondiale dans le département du Tarn. Guide des monuments, stèles et plaques commémoratives, s.l., Imprimerie nationale, s.d., 71 p.

Commission départementale de l'information historique pour la Paix, Sur les chemins de l'histoire et du souvenir. Les lieux de mémoire de la Seconde Guerre mondiale, département de la Saône-et-Loire, Mâcon, CDIHP, 1988, $304 \mathrm{p}$.

BARCELLINI (Serge) ; WIEVIORKA (Annette), Passant souviens-toi! Les lieux du souvenir de la Seconde Guerre mondiale en France, Paris, Graphein, 1999, $523 \mathrm{p}$.

PANICACCI (Jean-Louis), Les Lieux de mémoire de la Deuxième Guerre mondiale dans les Alpes-Maritimes, Nice, Éditions Serre, 1997, 143 p.

Sur les cheminots

AUZAS (Vincent), « Mémoires de la Résistance chez les cheminots », in AHICF, Une entreprise publique dans la guerre. La SNCF, 1939-1945, Paris, PUF, 2001, p. 323-330.

AUZAS (Vincent), «La mémoire de la résistance chez les cheminots : construction et enjeux, septembre 1944-novembre $1948 »$, mémoire de maitrise d'histoire, sous la dir. de Jean-Louis et Christian Chevandier, université Paris I, 2000, 182 p. [Déposé à l'AHICF.]

CHABOUD (Jack); DUPUIS (Dominique), Quai des bulles. Le train dans la bande dessinée, Paris, La Vie du rail, 1985, 222 p.

CHEVANDIER (Christian), « Les cheminots, la SNCF et la Seconde Guerre mondiale ", in AHICF, Une entreprise publique dans la guerre. La SNCF, 1939-1945, Paris, PUF, 2001, p. 305-321.

WOLIKOW (Serge), « Syndicalistes cheminots et images de la Résistance », in AHICF, Une entreprise publique dans la guerre. La SNCF, 1939-1945, Paris, PUF, 2001, p. 299-304.

* Autour du cinéma en général et de (La) Bataille du Rail en particulier

«La Bataille du Rail », numéro spécial de L'Avant-scène cinéma, n 442, (mai 1995).

ARMAND (Louis), Propos ferroviaires, Paris, Fayard, 1971, chap. 6, p. 131137.

BERTIN-MAGHIT (Jean-Pierre), «La Bataille du rail: de l'authenticité à la chanson de geste ", Revue d'histoire moderne et contemporaine, tome XxiI, n 2 (avril-juin 1986), nº spécial «Cinéma et sociétés », p. 280-300. 
BERTIN-MAGHIT (Jean-Pierre), « Les résistants du rail », Historia, n 575 (novembre 1994), p. 22-28.

CARRIÈRE (Bruno), «Les secrets de la Bataille du rail », La Vie du rail, $\mathrm{n}^{\circ} 2541$ (10 avril 1996), p. 42-47.

CLÉMENT (René) ; AUDRY (Colette), La Bataille du rail, Paris, Robert Laffont, 1949, 189 p.

DELAGE (André), "Les cheminots français dans La Bataille du rail», Chemins de fer, numéro spécial Noël 1947, 11 p.

Sous-inspecteur à la SNCF, habilité par la direction de l'entreprise pour suivre la réalisation du film.

IONASCU (Michel), «L'homme disparu : les cheminots dans le cinéma français », maitrise AES, sous la dir. de Myriam Tsikounas, université Paris I, 1994, $114 \mathrm{p}$.

IONASCU (Michel), " Cheminots argentiques : l'image d'un groupe social dans le cinéma et l'audiovisuel français ", thèse Recherches cinématographiques et audiovisuelles, sous la dir. de Michèle Lagny, université Paris III, 1999, 360 p.

IONASCU (Michel), Cheminots et cinéma. La représentation d'un groupe social dans le cinéma et l'audiovisuel français, Paris, L'Harmattan, 2001, 400 p.

LINDEPERG (Sylvie), «L'opération cinématographique. Équivoques idéologiques et ambivalences narratives dans $L a$ Bataille $d u$ rail», Annales histoire sciences sociales, juillet-août 1996/4, p. 759-779.

LINDEPERG (Sylvie), Les Écrans de l'ombre. La Seconde Guerre mondiale dans le cinéma français, Paris, Éditions du CNRS, 1998, 443 p.

WOLIKOW (Serge), « La Bataille du rail : la création d'une image collective de la résistance des cheminots », in « René Clément », L'Avant-scène cinéma, $\mathrm{n}^{\circ} 442$ (mai 1995), p. 74-77. 